\title{
Coulisses
}

Revue de théâtre

25 | Hiver 2002

Varia

\section{6, coopérative artistique, penser une économie de la fonction de l'artiste}

Entretien avec Jean Lambert-Wild. Propos recueillis par Lucile Garbagnati

Lucile Garbagnati

\section{CpenEdition}

Journals

Édition électronique

URL : http://journals.openedition.org/coulisses/5911

DOI : 10.4000/coulisses.5911

ISSN : 2546-9460

Éditeur

Presses universitaires de Franche-Comté

Édition imprimée

Date de publication : 1 janvier 2002

Pagination : 14-18

ISBN : 2-84627-052-X

ISSN : $1150-594 X$

Référence électronique

Lucile Garbagnati, «326, coopérative artistique, penser une économie de la fonction de l'artiste », Coulisses [En ligne], 25 | Hiver 2002, mis en ligne le 24 octobre 2019, consulté le 15 novembre 2019 URL : http://journals.openedition.org/coulisses/5911 ; DOI : 10.4000/coulisses.5911

Ce document a été généré automatiquement le 15 novembre 2019.

Coulisses 


\section{6 , coopérative artistique, penser une économie de la fonction de l'artiste}

Entretien avec Jean Lambert-Wild. Propos recueillis par Lucile Garbagnati

\section{Lucile Garbagnati}

coulisses. (c.) : Jean Lambert-Wild vous êtes connu comme auteur et comme metteur en scène. Votre pièce Orgia, dont Coulisses $n^{\circ} 24$ a rendu compte, a créé l'événement lors la saison 2000-2001. Elle est reprise actuellement au Théâtre de la colline, à Paris. Vous êtes en cours de création d'une forme de théâtre opératique originale en multi résidence: Spaghetti club. Vous êtes également le directeur artistique de 326, coopérative artistique. Lorsqu'on est jeune et déjà reconnu, pourquoi avoir voulu fonder "une coopérative artistique »?

Jean Lambert-Wild (J. L.-W.) : Cette fondation provient d'un constat douloureux, de la disparition "des miens ", d'artistes dont je me sens proches. Ils cessent d'exister soit par la mort physique, soit par la lente agonie de leurs facultés de création, du fait de conditions économiques insoutenables, soit par le renoncement. Il s'agit de prendre en charge une partie de la solitude physique dans laquelle se trouve l'artiste, c'est-à-dire les difficultés dans lesquelles il se débat pour faire face aux exigences administratives et économiques de toute demande de financement. En effet, le spectacle vivant nécessite une structuration forte, à laquelle bien des artistes sont incapables d'accéder soit par manque de moyens, par ignorance voire par coquetterie. Il devient donc indispensable de s'associer avec d'autres si on veut trouver des solutions. Chaque année la France compte $10 \%$ d'artistes en plus, c'est-àdire de personnes qui relèvent $d u$ statut d'intermittents. On peut, d'ailleurs, s'interroger sur les raisons d'un tel foisonnement. La réflexion de Guy Debord peut être utile; on est artiste parce qu'on ne veut pas aller à l'usine, se soumettre à la discipline d'un chef quel qu'il soit. Cela repose sur l'idée fausse que l'art est facile. La mise en place d'un système de formation reproductif, comme celui de l'Éducation nationale avec les classes A3 et les formations universitaires en facilite la 
propagation. Il y a également l'éclat de la star. Il s'agit de penser une économie de la fonction de l'artiste en luttant d'abord contre la vanité. Quand quelque chose ne va pas tout le monde accuse l'institution. En fait, cette dernière applique ce que les artistes préconisent, elle est dans la continuité d'une logique artistique. Les artistes sont au pouvoir. Mais, soit ils ne communiquent pas, soit ils sont dans un rapport de compétition stérile. Sans doute ont-ils peur de la spoliation, et du qu'en dira-t-on. Il peut exister dans le milieu un climat de cynisme et de méchanceté, qu'on a du mal à imaginer quand on n'y appartient pas. Pour ma part, je suis privilégié, puisque Henri Taquet, directeur du théâtre Le Granit, scène nationale de Belfort, m'a fait une totale confiance en m'accordant une résidence, mais tout le monde n'a pas cette chance. D'où l'idée de fonder une coopérative dans l'idée de partage et d'aide mutuelle : tu es aidé aujourd'hui quand tu en as besoin, et tu aideras demain ceux qui seront dans la même situation. Elle constitue aussi un garde-fou à l'ego de par les réflexions sur la fonction de l'art, l'apprentissage et la responsabilisation économique de chacun de ses membres. La coopérative s'est aussi constituée autour d'un projet artistique qui est "de réunir des artistes de différentes disciplines couvrant un champ artistique le plus étendu et le plus varié possible». Il s'agit «d'interroger et d'expérimenter dans un esprit de coopération et de réflexion de nouvelles associations esthétiques dont les connections bouleverseront le cadre habituel des conventions de narration et de représentation de chaque discipline." Il s'agit de renouveler les rapports d'échanges et de travail entre artistes en les fédérant «sans imposer un dogme ou un manifeste.»

L'action de la coopérative peut se concrétiser par:

- « des projets collectifs qui font appel à toutes les énergies de la coopérative

- des projets individuels soutenus par une partie des membres de la coopérative,

- par le soutien apporté aux artistes émergents et désireux de rejoindre ou de partager les intérêts de la coopérative. "

Les membres fondateurs témoignent justement de cette orientation: Jean-Luc Therminarias, compositeur associé au Centre national de création musicale de Marseille, Renaud Lagier, éclairagiste, Christophe Balgero écrivain et vidéaste, Christophe Farion, régisseur. 


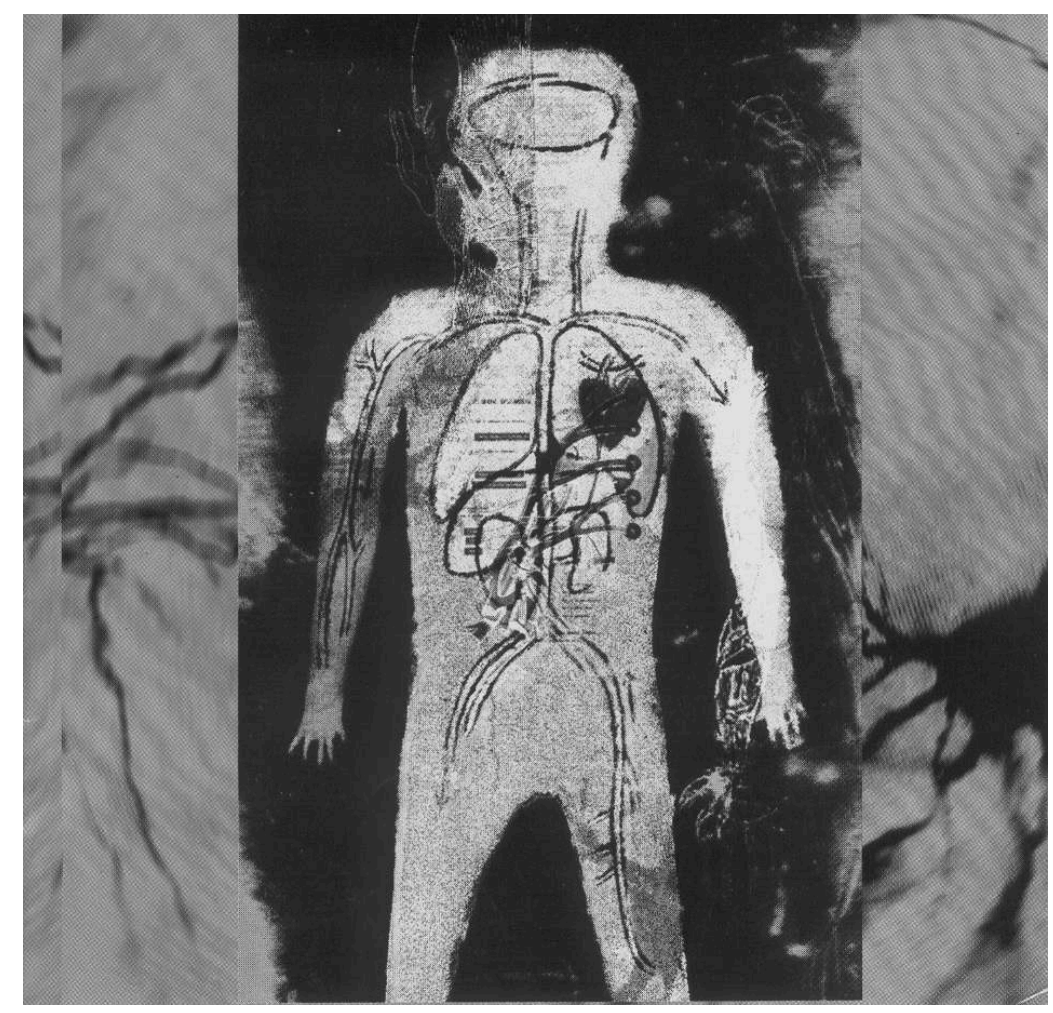

Ersellia Ferron

\section{C. : QUELS SONT VOS RÉALISATIONS ET VOS PROJETS ?}

J. L.-W. : Les réalisations sont indissociables des projets, ne serait-ce qu'au niveau économique coproduction et diffusion forment un tout. Il ne faut pas oublier que la coopérative accueille toutes les formes d'art. Pour les formes théâtrales de nos activités, Orgia est programmé au Théâtre de la colline du 10 janvier au 15 février, et au Centre dramatique d'Orléans du 27 au 29 mars 2002. Le Terrier d'après Franz Kafka, théâtre en appartement, est à la disposition de tous ses hôtes potentiels de janvier à juin 2002. Aphtes, performance pour acteur, petite cuillère et vidéo va être reprise dans différents festivals et musées d'art contemporain. Nous proposons la Randonnée théatrolopoéticopédestre, qui avait connu un véritable succès l'an dernier, avec, bien sûr, un programme différent. Les créations portent moins sur le théâtre que sur d'autres propositions artistiques défendues par nos membres. Des performances: Crise de nerfs, à propos de l'enfermement et de la patience pour acteur scaphandrier, Élégie à Mathilde Petit, performance vidéo et poétique qui sera présentée dans des forts des régions Franche-Comté, Rhône-Alpes et Provence Alpes Côte d'Azur, Le Mur, performance pour acteur et mur sonorisé à propos des chocs et des violences du renoncement poétique de l'homme moderne. Nous créons aussi des concerts de musique contemporaine tant au théâtre Granit, qu'au Théâtre de la colline qu'au Festival du G.M.E.M. "Les musiques d'aujourd'hui à Marseille». Nous mettons en place sur le Web une exposition de Ersellia Ferron, plasticienne/ performeuse new-yorkaise. Nous la compléterons, en septembre 2002, par une galerie virtuelle de ses travaux. Par la suite, elle s'ouvrira à d'autres artistes. Les ateliers nous permettent une démarche de type pédagogique. Il peut s'agir de toucher un public autre comme dans celui qui est mené pour la deuxième année consécutive avec le $35^{\mathrm{e}}$ régiment d'infanterie de Belfort, soit d'approfondir une réflexion où les esprits 
épris de résistance peuvent se rassembler, élargir le champ de leurs pensées, et assouplir le mouvement de leur jugement comme dans l'atelier critique. Nous mènerons à bien le projet de Spaghetti's club, expérience d'écriture poétique et musical, qui, sur deux ans et en sept étapes aura exploré des formes d'art différentes, dans des lieux différents et des acteurs différents. Les étapes à venir sont révélatrices du projet. L'étape $n^{\circ} 5$, Le point de vue de Lewis Carroll, pièce musicale avec quatre acteurs anglais, aura lieu à Londres en mars 2002. L'étape $n^{\circ} 6$, Le point de vue de Giordano Bruno consistera en un atelier d'écriture et un atelier musical avec les détenus et les surveillants de la maison d'arrêt de Belfort. L'étape $\mathrm{n}^{\circ}$ 7, La conclusion, créée au cours de l'automne 2002, rassemblera en un seul moment l'ensemble des travaux menés dans toutes les Étapes. Cependant la complexité technique et artistique de ce projet oblige à anticiper très en amont les répétions. Des expérimentations musicales, scénographiques et textuelles auront donc lieu d'avril à juin 2002. Nous sommes également éditeurs de disques, 326 musique, label de musique contemporaine. Aux deux disques déjà édités, nous prévoyons d'en ajouter sept autres.

\section{C. : TOUTE CETTE ACTIVITÉ REPRÉSENTE UN TRÈS gROS INVESTISSEMENT PERSONNEL.}

J. L.-W.: C'est monstrueux en terme d'écoute et aussi du principe d'abandon du pouvoir. La question de l'artiste pose celle du rapport au pouvoir. Au théâtre, le metteur en scène est le leader, qui peut se considérer comme un artiste, ou comme un patron. Mais c'est au pied du mur que l'on voit le maçon. En fait, il s'agit de mettre en place un protocole d'échanges, qui implique un engagement et une certain militantisme minimum. Je suis actuellement au poste clé comme administrateur et comme économe. En effet, du fait des soutiens dont je bénéficie, je peux porter la coopérative. Il faut que le travail des uns profite à tous. L'expérience de Spaghetti's club est tout à fait passionnante. On dépasse à la fois les frontières géographiques, artistiques et mentales. On constate que tous les enjeux artistiques sont dans la durée pour trouver la forme adéquate, qui peut bouleverser le mode de travail et l'entourage. Il s'agit en fait de ne pas réunir seulement des actions mais aussi des volontés. La coopérative considère qu'économique peut aller de pair avec éthique. Elle peut être fondée sur l'écoute, l'entente, l'intérêt réciproque. L'honnêteté consiste à remplir ses engagements. Les membres sont ceux qui désirent se confronter à ces échanges, qui refusent une carrière en solo. Le projet s'inclut dans une réflexion plus vaste qui est celle de la fonction de l'artiste dans la cité: comment créer des synergies productrices d'intérêt, d'intelligence, de sens. Autrement dit, qu'est-ce qu'un théâtre de service public ? Comment utiliser au mieux l'argent de la collectivité nationale? Il s'agit aussi de dépasser les jugements des médiocres au profit du dépassement de soi pour l'aboutissement du projet. Nous sommes en train d'en vivre une étonnante illustration. Comme vous les savez, nous animons des ateliers au $45^{\mathrm{e}}$ R.I de Belfort, le lieutenant-colonel a pris sa retraite pour nous rejoindre en tant qu'administrateur !!!

\section{C. : D'OÙ VOUS VIENT CETTE CONCEPTION ?}

J. L.-W. :Je la dois à ceux que j'appellerai mes maîtres : mon père, qui m'a fait confiance, mon instituteur de CM2, mes professeurs de philosophie et de latin qui m'ont appris que le travail et la rigueur n'enlèvent pas la joie, bien au contraire, et révélé la puissance de Dyonisos. J'ai découvert le théâtre à l'occasion de la représentation des Trois sœurs de Tchékhov, mise en scène de Mathias Langhoff. Ce 
fut un choc. Je lui ai donc demandé un rendez-vous. J'ai passé des heures, des jours à faire le pied de grue devant sa porte, jusqu'au moment où il m'a accepté. C'est avec lui que j'ai appris le métier ainsi qu'avec sa collaboratrice, puis avec Michel Dubois, alors au C.D.N. de Caen, puis avec Michel Simenot, et Henri Taquet.

c. : Puisque vous êtes en résidence au Granit, que vous a apporté, Henri Taquet ?

J. L.-W. : À structurer l'emballement de ma pensée. À envisager l'équilibre des droits et des devoirs. Trop de projets tuent les projets. Il m'a évité la folie. Je crois au travail, on ne travaille pas assez, un artiste doit baigner dans la sueur, parce que travailler c'est s'éduquer. La télévision, par son effet hypnotique contribue à l'abrutissement de tout un pays, et lui enlève tout désir. Elle lui offre un choix dans le rien, entre quinze programme plus ou moins identiques. En supprimant cette envie, la société fabrique des névroses. Elle est cause de l'actuelle épidémie de dépression, qu'elle soit individuelle ou collective, qu'elle relève du domaine économique, social ou politique. La coopérative 326 offre aux artistes qui ont de l'orgueil, c'est-à-dire, un fort sentiment de leur valeur, le temps pour travailler, pour faire quelque chose de cette impérieuse nécessité qui les hante.

Contact : 4 rue du président Roosevelt, 90000 Belfort, Tel 0384222649 / Fax : 038422 74 95. email : lambert-wild@wanadoo.fr Pour les disques, site internet de vente online : www.326music.com 